\title{
Surface Ionization on Metal Oxide Gas Sensors
}

\author{
A. Ponzoni ${ }^{1}$, D. Zappa ${ }^{1,2}$, E. Comini ${ }^{1,2}$, G. Faglia ${ }^{1,2}$, G. Sberveglieri ${ }^{1,2}$ \\ ${ }^{1}$ CNR-IDASC SENSOR Lab, Via Branze 45, 25123 BRESCIA, Italy \\ Corresponding author's e-mail address: andrea.ponzoni@ing.unibs.it \\ ${ }^{2}$ University of Brescia, Chem. and Phys. Dept., Via Valotti 9, 25133 Brescia, Italy
}

\begin{abstract}
:
In this work we present the development of surface ionization (SI) gas sensors with planar layout based on bundles of nanorods. We exploit a simple method, compatible with planar deposition techniques, to integrate the layer of nanorods into the functional device. The comparison between the same device provided and not provided with the nanorod layer shows that the measured signal arises from nanorods and not from the Pt electrodes. We further show that the adopted configuration and the exploitation of the nanorod morphology allow to reduce the working temperature from $600-900{ }^{\circ} \mathrm{C}$ to $300-400{ }^{\circ} \mathrm{C}$ and the voltage bias form $10000 \mathrm{~V} / \mathrm{cm}$ to $1500 \mathrm{~V} / \mathrm{cm}$ with respect to the traditional vertical layout.
\end{abstract}

Key words: Nanowires; Metal Oxide; Surface Ionization; Chemiresistors

\section{Introduction}

Metal oxide gas sensors have been widely studied in literature and exploited at industrial level thanks to their high sensitivity to a broad range of chemical compounds, the cheap methods of fabrication and the compatibility with silicon technology. Furthermore, their reduced weight, size and power consumption, which allows the development of portable instrumentation and/or the use in sensor networks.

Beside these potentialities, metal oxides suffer the reduced selectivity and not yet satisfactory long time stability.

Concerning selectivity, the most adopted solution is the electronic nose system: a sensor array with a pattern recognition software handling its collective response. To increase the variety of metal oxides that can be exploited in such systems, different approaches have been developed, such as, for example, materials based on doped, catalyzed or mixed oxides [1]. Other focused on the development of materials with controlled morphologies such as layers with pore size enabling the selective diffusion of molecules [2] or single crystalline nanowires [3, 4].

Recently, approaches based on novel sensing mechanisms, namely surface ionization, have been considered to exploit the potentialities offered by nanotechnology and nanostructured materials.

Surface ionization (SI) consists in the formation of positive and negative ions in the course of thermal desorption of molecules [5]. This working principle was traditional exploited in a vertical configuration, using a warm material (metallic or a metal oxide), where the gas adsorbs and ionizes, and a high electric field to extract ions and accelerate them toward a suspended electrode. High 
temperatures, usually between 600 and 900 ${ }^{\circ} \mathrm{C}$, and high electric fields, around 10000 $\mathrm{V} / \mathrm{cm}$, are necessary to activate these processes [5, 6]. Enhanced sensitivity has been observed using metal oxide layers with nanowire (nanorod) morphology, ascribing such an improvement to the nanowire structure, namely their high crystalline quality and their nanosized diameter, which ease the concentration of high electric field at their surfaces for ion extraction [6].

SI devices based on single nanowires have also been developed exploiting a planar layout with the single nanowire facing a Pt counterelectrode [7]. Results showed that this configuration can work at reduced temperature and at lower voltages with respect to traditional SI devices; nevertheless, it requires the use of advanced lithographic techniques such as Focused Ion Beam (FIB) to properly contact the single $\mathrm{SnO}_{2}$ nanowire and is thus hardly exploitable at an industrial level. So far, strategies overcoming this technological gap are still need. In the present work we present our results on the development of SI devices based on horizontal layout but using a preparation method suited to directly deposit the oxide layer (based on a bundle of nanowires) over the functional substrate.

\section{Experimental}

In this work we focused on $\mathrm{CuO}$ nanorods.

Nanorods were prepared using a simple thermal oxidation method, consisting in a controlled oxidation of a thin copper layer inside a tubular furnace in a controlled atmosphere. This method feature the advantage of easing the selective growth the nanorods, just pattering (for example with shadow masking technique) the deposition of the metal film. The nanorods grow only where there is the copper layer. The thin metal copper layer is deposited via sputtering from a copper target. As expected the sputtering time has effect on the layer thickness, but we find out that the sputtering temperature changes the morphology of the copper metal layer and has effect also on the nanorods growth. After the thin layer deposition, samples are cleaned in air plasma for $5^{\prime}$ at $15 \mathrm{~W}$, to remove the very thin copper oxide layer that it's always present due to the reactivity of copper in air. After the plasma etching, samples were oxidized inside a tubular furnace for 15 hours at the temperature of $400{ }^{\circ} \mathrm{C}$ in an atmosphere composed by $80 \% \mathrm{O} 2$ and $20 \% \mathrm{Ar}$.
The morphology of the obtained $\mathrm{CuO}$ layers is shown in Fig. 1. The $\mathrm{CuO}$ (Tenorite) phase was identified through XRD measurements.

Sensors were prepared using a $2 \mathrm{~mm} \times 2 \mathrm{~mm}$ alumina substrate, according to the layout shown in Fig. 2, where the L-shaped electrodes are used to bias the oxide layer and the $\mathrm{Pt}$ meander is grounded and used as counter-electrode.

In order to identify the measured current as an effective ionization current, additional sensors, with the same structure but not featuring the oxide layer, were also prepared. In the following, $\mathrm{CuO}$ and $\mathrm{Pt}$ will be used to refer to the former and the latter device respectively.

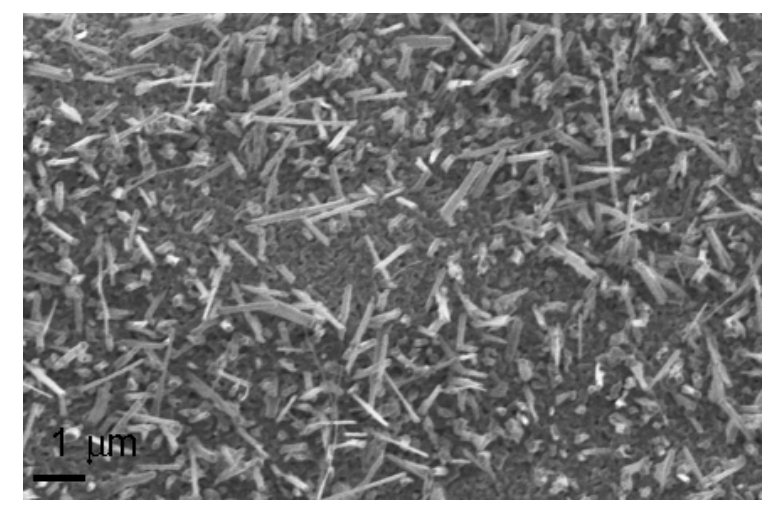

Fig. 1. SEM image of the CUO nanowire layer.

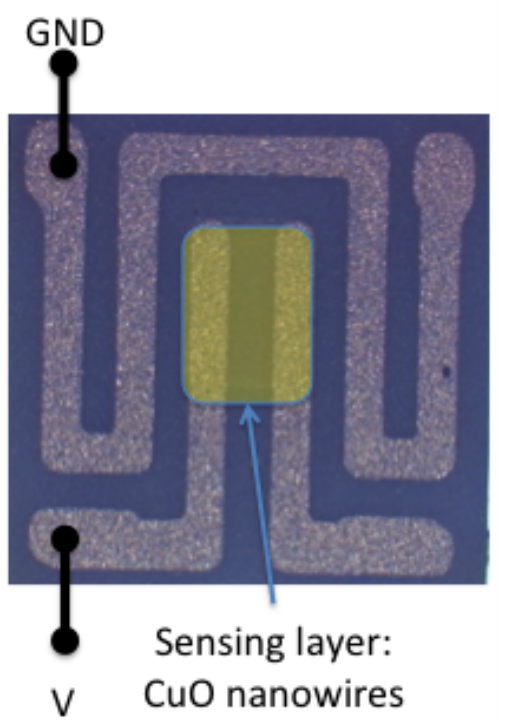

Fig. 2. Sensor layout for surface ionization measurements with planar configuration.

Gas sensing measurements were carried out in a stainless steel chamber under controlled humidity and temperature conditions. Atmosphere composition was controlled by 
means of mass flow controllers balancing flows coming from certified bottles.

\section{Results}

The first step to develop SI devices based on planar layout is to prove that the signal comes from surface ionization phenomena instead of leakage current or other undesired effects.

To this aim, the sensor temperature was tested in the range $200-550{ }^{\circ} \mathrm{C}$ and the bias voltage in the range 1-300 $\mathrm{V}$ (corresponding to an electric field of 50-15000 V/cm).

Despite results obtained with $\mathrm{SI}$ devices designed according to the vertical configuration can provide a useful reference, phenomena in planar configurations are likely to be much more complex. In a planar configuration, both electrodes are heated at the same temperature (while in the vertical layout the counter-electrode is suspended over the heated device and is thus at a lower temperature), so that they can be both active in producing SI currents. Also, with a planar layout, ions generated over the electrode surface does not travel in air to reach the opposite electrode but are more likely to travel over the substrate surface.

So far, SI currents have been identified looking for asymmetries with respect to the bias voltage, which should arise in the $\mathrm{CuO}$ sensor (due to the facing of $\mathrm{CuO}$ and $\mathrm{Pt}$ electrodes) and not in the Pt sensor (due to the facing of two Pt electrodes).

As an example, responses to acetone and ethanol obtained at the senor temperature of $350{ }^{\circ} \mathrm{C}$, with a voltage bias of $+30 \mathrm{~V}$ are shown in Fig. 3 (a). The same measurements have been repeated with a bias of $-30 \mathrm{~V}$, Fig. 3 (b).

It is evident for these results that when the $\mathrm{CuO}$ sensor is biased with $+30 \mathrm{~V}$, it exhibits reliable responses to acetone (response amplitude increases with increasing the gas concentration). Differently, with a negative bias it shows some weak oscillations that disappear with time. The Pt sensor does not exhibit reliable responses, neither when positively nor when negatively biased. In both cases, it shows weak oscillations, with amplitude not related to the gas concentration, suggesting that $\mathrm{SI}$ is not activated in this device. The Pt layer feature indeed a compact structure that requires a much higher temperature and electric field to activate the SI signal with respect to layers with nanorod morphology [6].
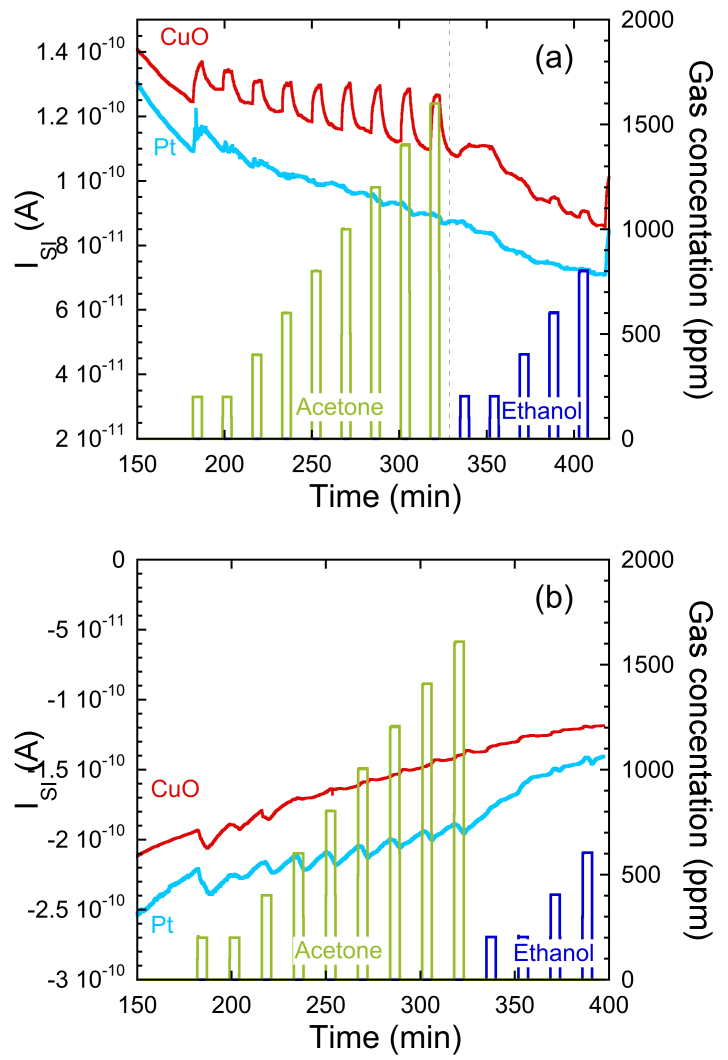

Fig. 3. Dynamic response of the $\mathrm{CuO}$ and $\mathrm{Pt}$ sensors to different concentrations of acetone and ethanol. Results have been obtained heated devices at the sensor temperature of $350{ }^{\circ} \mathrm{C}$ and using a bias of $+30 \mathrm{~V}(\mathrm{a})$ and $-30 \mathrm{~V}(\mathrm{~b})$.

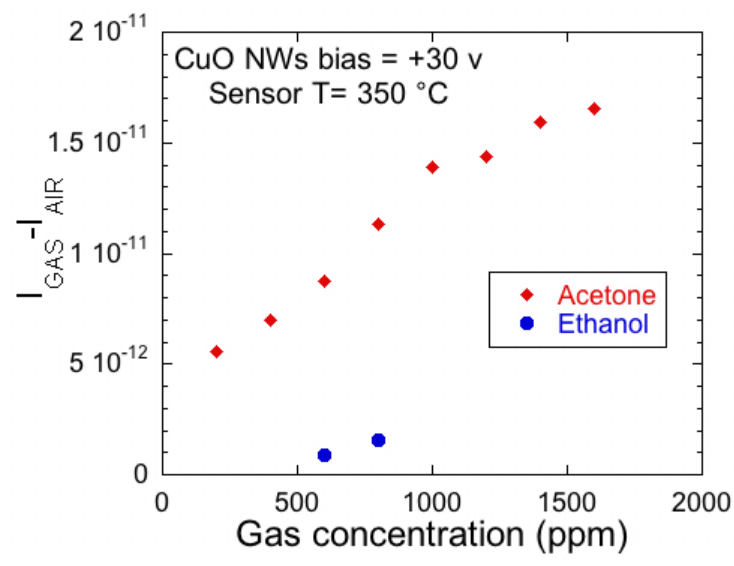

Fig. 4. Calibration curve of the $\mathrm{CuO}$ sensor to acetone and ethanol.

The response of the $\mathrm{CuO}$ sensor to different concentrations of acetone and ethanol are summarized in Fig. 4, showing the sensor calibration curve to these compounds. The much higher sensitivity to acetone than to 
ethanol can be reasonably ascribed to its lower ionization energy $(9.70 \mathrm{eV})$, compared to the $10.48 \mathrm{eV}$ of ethanol, [8].

It's worth nothing that with the planar configuration, the working temperature and the

\section{Conclusions}

In this work we've presented a method based on the deposition of a metallic layer and its further oxidation to obtain metal oxide nanorods, showing in particular the suitability of the technique to prepare SI ionization devices with planar layout. Focusing on $\mathrm{CuO}$ nanorods, we've further shown that the nanorod layer is fundamental to produce the SI signal and calibrated the device toward two common chemicals such as acetone and ethanol.

These results are promising for the development of hybrid electronic nose systems, where the exploitation of different sensing mechanism, namely redo-ox reactions in chemiresistors and surface ionization phenomena in SI devices, would enhance the selectivity capability of the instrument.

\section{Acknowledgements}

The research leading to these results has received funding from the European Community's 7th Framework Programme, under the grant agreement $n^{\circ} 247768$, and from the Russian Federation Government, under the State Contract 02.527.11.0008, within the collaborative Europe-Russia S3 project. voltage bias can be much lowered with respect to the vertical configuration. Optimal performance are here obtained at the sensor temperature of about $350{ }^{\circ} \mathrm{C}$ and with an electric field of about $1500 \mathrm{~V} / \mathrm{cm}$.

\section{References}

[1] Sberveglieri G., Recent developments in semiconducting film gas sensors, Sens. Actuator B-Chem. 23, 103-109 (1995); doi: 10.1016/0925-4005(94)01278-P

[2] Kanan S.M., Waghe A., Jensen B.L., Tripp C.P., Dual WO3 based sensors to selectively detect DMMP in the presence of alcohols, Talanta 72 , $401-407 \quad(2007) ; \quad$ do i : 10.1016/j.talanta.2006.10.046

[3] Zhang D., Liu Z., Li C., Tang T., Liu X., Han S., Lei B., Zhou C., Detection of NO2 down to ppb levels using individual and multiple In2O3 nanowire devices, Nano Lett. 4, 1919-1924 (2004); doi: 10.1021/nl0489283

[4] Ponzoni A., Baratto C., Bianchi S., Comini E., Ferroni M., Pardo M., Vezzoli M., Vomiero A., Faglia G., Sberveglieri G., Metal oxide nanowire and thin-film-based gas sensors for chemical warfare simulants detection, IEEE Sens. J. 8, $735-742 \quad(2008) ; \quad$ do i : 10.1109/JSEN.2008.923179

[5] Rasulev U. Kh., Zandberg E. Ya., Surface Ionization of Organic Compounds and its Applications, Progr. Surf. Sci. 28, 181-412 (1988); doi: 10.1016/0079-6816(88)90003-2.

[6] Hackner A., Habauzit A., Müller G., Comini E., Faglia G., Sberveglieri G., Surface ionization gas detection on platinum and metal oxide surfaces, IEEE Sens. J. 9, 1727-1733 (2009); doi: 10.1109/JSEN.2009.2030705

[7] Hernandez-Ramirez, F., Prades, J.D., A. Hackner, T. Fischer, G. Mueller, S. Mathur, J.R. Morante, Miniaturized ionization gas sensors from single metal oxide nanowires, Nanoscale 3, 630-634 (2011); doi: 10.1039/c0nr00528b

[8] http://webbook.nist.gov/chemistry/ 